\title{
Customised Cytoarchitectonic Probability Maps Using Deformable Registration: Primary Auditory Cortex
}

\author{
Lara Bailey ${ }^{1}$, Purang Abolmaesumi ${ }^{1,3}$, Julian Tam ${ }^{1}$, Patricia Morosan ${ }^{4}$, \\ Rhodri Cusack $^{7}$, Katrin Amunts ${ }^{4,5,6}$, and Ingrid Johnsrude ${ }^{2}$ \\ ${ }^{1}$ Department of Computer Science, Queen's University, Canada \\ ${ }^{2}$ Department of Psychology, Queen's University, Canada \\ ${ }^{3}$ Department of Electrical and Computer Engineering, Queen's University, Canada \\ ${ }^{4}$ Institute of Medicine, Research Center Juelich, Juelich, Germany \\ ${ }^{5}$ Department of Psychiatry and Psychotherapy, RWTH University Aachen, Germany \\ ${ }^{6}$ Brain Imaging Center West (BICW), Germany \\ 7 MRC Cognition and Brain Sciences Unit, Cambridge, England \\ purang@cs.queensu.ca
}

\begin{abstract}
A novel method is presented for creating a probability map from histologically defined cytoarchitectonic data, customised for the anatomy of individual fMRI volunteers. Postmortem structural and cytoarchitectonic information from a published dataset is combined with high resolution structural MR images using deformable registration of a region of interest. In this paper, we have targeted the three sub-areas of the primary auditory cortex (located on Heschl's gyrus); however, the method could be applied to any other cytoarchitectonic region. The resulting probability maps show a significantly higher overlap than previously generated maps using the same cytoarchitectonic data, and more accurately span the macroanatomical structure of the auditory cortex. This improvement indicates a high potential for spatially accurate fMRI analysis, allowing more reliable correlation between anatomical structure and function. We validate the approach using fMRI data from nine individuals, taken from a published dataset. We compare activation for stimuli evoking a pitch percept to activation for acoustically matched noise, and demonstrate that the primary auditory cortex (Te1.0) and the lateral region Te1.2 are sensitive to pitch, whereas Te1.1 is not.
\end{abstract}

\section{Introduction}

The increased spatial resolution of functional magnetic resonance imaging (fMRI) permits researchers to localize neural activity with respect to an individual subject's anatomy. In order to draw general conclusions about the relationship between brain structure and function, it is generally necessary to pool results across several subjects. This is usually done by performing an additional image registration, which aligns each subject's data to a standard brain template (e.g., the ICBM152 template). This additional 'normalisation' step also allows results to be compared among studies.

N. Ayache, S. Ourselin, A. Maeder (Eds.): MICCAI 2007, Part II, LNCS 4792, pp. 760 768. 2007.

(C) Springer-Verlag Berlin Heidelberg 2007 
Analysing functional data across subjects usually involves assessing the magnitude of brain activity at the voxel level, but high inter-subject variability makes it difficult to accurately achieve a good match from subject to subject. Consequently, the region of pooled activation across subjects is diffused, and for small regions, statistical analysis may not reveal significant activation. The common solution is to apply spatial smoothing to each subject's functional data. Smoothing not only increases the overlap of activation among subjects and thus the significance of the results, but also decreases the spatial resolution.

Improving registration for gross anatomical features across subjects is not enough, since such features are not a reliable indicator of functionally distinct areas ([2, 3]). Instead, microanatomical features such as cytoarchitecture are more directly linked to functional regions (4], [5]) should be used to increase the registration accuracy and thus decrease the need for spatial smoothing. Unfortunately it is currently not possible to observe microanatomical structures in routine magnetic resonance (MR) images acquired from human volunteers for most cortical regions. A solution to this dilemma may be found in the use of probability maps of microanatomical regions, derived from histological measurements in postmortem samples. In such maps, the value at each voxel indexes the likelihood of that voxel belonging to a particular cytoarchitectonic region. Such maps, coregistered with the anatomy of each fMRI volunteer, can be used as weighted filters on fMRI data, obviating any need for traditional inter-subject spatial averaging and isotropic smoothing, and permitting functional differentiation of small, adjacent, brain regions.

\section{$1.1 \quad$ Previous Work}

Quantitative cytoarchitectonic analysis was conducted on brain sections stained for cell bodies, from ten postmortem human brains. An observer-independent method was used to determine areal borders [1, [7, and [8]. The results were digitized and mapped onto high-resolution structural magnetic resonance images, which had been previously acquired on each postmortem specimen, creating 3D cytoarchitectonic volumes. This work reveals three 'primary-like' auditory regions, Te1.0, Te1.1, and Te1.2, overlapping with Heschl's gyrus in each hemisphere; these are distinguished on the basis of cell architecture across the six layers of cortex (see Fig 1, 6, 9]). By 1) registering the postmortem structurals and cytoarchitectonic datasets to match a normal brain template (see Fig. 2(a) step 1), and then 2) averaging the registered cytoarchitectonic datasets (see Fig. 2(a) step 2), a probability map was created for each cytoarchitectonic area [6].

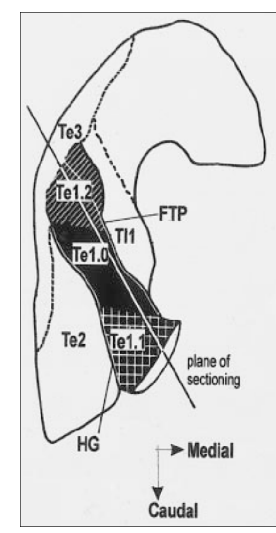

Fig. 1. Topography of cytoarchitectonically defined areas Te 1.0, 1.1, 1.2, Te2, TI1, and $\mathrm{Te} 3$ upon the (left) superior temporal plane in humans (From 6]) 


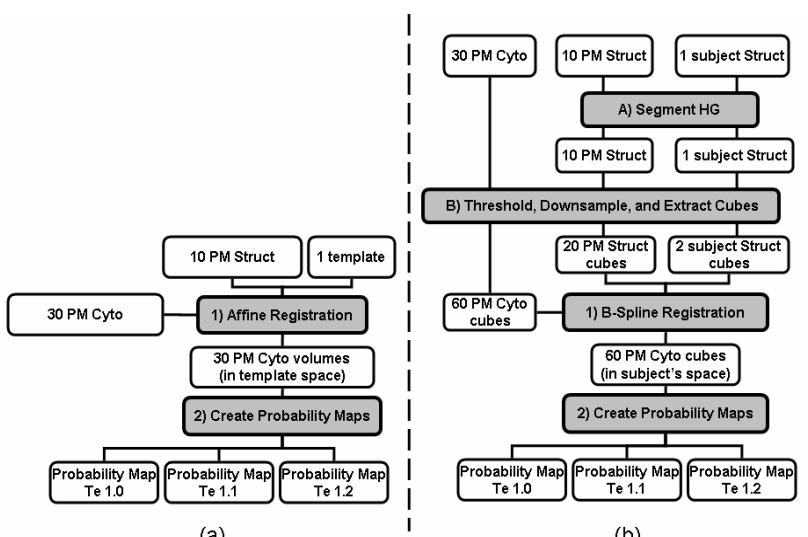

(a)

(b)

Fig. 2. Overview of (a) original and (b) proposed probability map generation process for auditory cortex (areas Te1.0, Te1.1, Te1.2)

\subsection{Procedure}

Probability maps tailored to the anatomy of individual fMRI subjects can be created by registering each cytoarchitectonic dataset directly to each fMRI subject's anatomy, and then averaging the registered datasets. We can use deformable registration algorithms, which produce highly detailed alignment among subjects (but which are computationally intensive) if we restrict our registration efforts to a small volume surrounding the region of Heschl's gyrus (HG) (Fig. 3(a) . The presented method of probability map generation can be used for any of the areas for which there is detailed cytoarchitectonic information coregistered with structural MR data from the same postmortem subjects, such as the data from the Jülich/Düsseldorf series, which is the most comprehensively studied, and largest, dataset of its kind in the world (see [2, 8, for review).

We first compare the anatomical precision and extent of the previously published probability maps [6] registered to a standard brain (Colin27) to maps created by using our high-dimensional ROI registration to the same standard brain. We then use fMRI data taken from a published study (Patterson et al. 2002) and use both the custom and published probability maps as weighted filters to investigate fMRI activation in each of the three cytoarchitectonic auditory regions defined in Section 1.1. We compare activation when subjects hear a noise stimu-

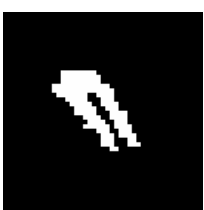

(a) $\mathrm{HG}$

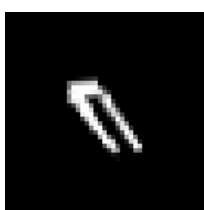

(b) Te 1

Fig. 3. (a) Grey matter of Heschl's Gyrus from gross anatomy, and (b) cytoarchitectonically defined Te1 from histological analysis lus with sufficient temporal structure to yield a pitch (which is fixed at one value for the duration of a particular stimulus, but varies over the course of the experiment), with an acoustically 
matched noise stimulus without temporal structure (and without pitch). This contrast is known to yield bilateral activity in lateral HG [1, but previous analysis did not assess whether this pitch-related activation was located in a specific cortical field, or whether it is laterally asymmetric.

\section{Method}

The proposed method of custom probability map generation is demonstrated in Fig. 2(b). Section 2.1 (steps A and B in Fig. 2(b)) describes how the region of Heschl's gyrus was preprocessed and extracted from both the high-resolution $(1 \times 1 \times 1 \mathrm{~mm})$ postmortem and the live subject's $(1 \times 1 \times 1 \mathrm{~mm}$ or $2 \times 2 \times 2 \mathrm{~mm})$ structural MR data, while Section 2.2 (step 1) describes the registration step used to align the extracted postmortem cubes to the subject's cubes. Finally, Section 2.3 (step 2) describes how the registered cytoarchitectonic data is averaged to create the subject's custom microanatomical probability map, which can then be used for fMRI analysis, as described in Section 2.4.

\subsection{Preprocessing}

Heschl's gyrus (HG) was 'painted' on each of the postmortem and fMRI subject's structural volumes using the criteria of Penhune et al. [10]. The grey-matter (GM) segmentation for the in-vivo structurals was obtained using SPM's 'segment' function [11, while the intersection of the cytoarchitectonic data with the painted gyrus defined the postmortem GM segmentation. The segmented volumes in standardized stereotaxic space were then thresholded, downsampled, and a cube surrounding the segmented HG in each hemisphere was extracted. Cubes at the same coordinates were also extracted from the three corresponding cytoarchitectonic volumes defining the sub-areas of Te1.

\subsection{Registration}

Each of the extracted postmortem structural ROIs were registered to the invivo structural ROIs using Insight ToolKit's (ITK) BSplineDeformable registration, with a grid size of 10x10x10. The generated non-linear transformation parameters were then applied to the cytoarchitectonic datasets of each subject, producing cytoarchitectonic datasets all registered to the in-vivo subject.

\subsection{Probability Map Generation}

A probability map for each of the three sub-regions of Te1 (Fig. 1) was created by summing the registered cytoarchitectonic cubes from each of the ten postmortems for that sub-region:

$$
p(i, v)=\frac{\sum_{q} \dot{p}(q, i, v)}{n I}
$$


where $p(i, v)$ is the probability of region $i$ containing voxel $v, \dot{p}(q, i, v)$ is the probability of region $i$ containing voxel $v$ for postmortem $q$ (grey-level of cytoarchitectonic volume), and $n$ is the number of postmortems. Since the warping is non-linear, it is possible for multiple voxels to be transformed to the same destination voxel. Thus, the three previously independent Te regions have the potential to intersect as a result of registration; this was corrected using the intersection factor $I=\sum_{k}\lceil p(k, v)\rceil$. The probabilistic ROIs were then re-inserted into stereotaxic space to create tailor-made probability maps for the in-vivo subject.

\section{4 fMRI Analysis}

The methods described in Sections 2.1 2.3 were used to create custom probability maps for each of nine fMRI participants [1, and then combined with their recorded fMRI activation using the following summary measure $s(i)$ :

$$
s(i)=\frac{\sum_{v} p(i, v) a(v)}{C(i)}
$$

where $p(i, v)$ is the probability of region $i$ containing voxel $v, a(v)$ is the activation at voxel $v$, and $C(i)=\sum_{v} p^{2}(i, v)$ is the normalisation constant for region $i$. Normalisation is necessary to ensure the summary statistic is scaled into units of "activation per voxel" and is no longer biased by the degree of variability among regions. This weighted summary measure was computed for each region (left and right hemisphere separately) in each subject, using both the custom probability maps and the previously published probability maps [6] normalized to a standard brain.

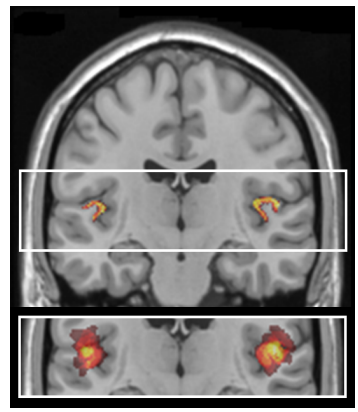

(a) Te 1.0

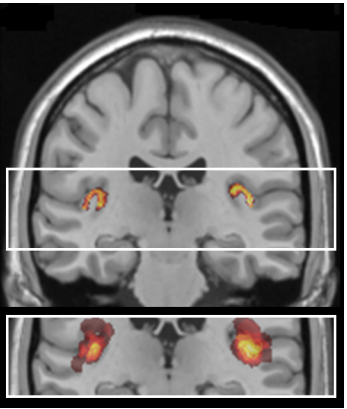

(b) Te 1.1

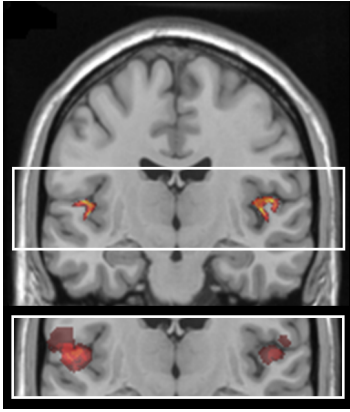

(c) Te 1.2

Fig. 4. Probability maps generated (top) and corresponding section of previous 6] probability maps (bottom), superimposed on the Colin27 structural 


\section{Results}

We compared the accuracy of our registration method with previous results [6] also in Colin27 space, by comparing the area covered by the two sets of probability maps. The maximum span of the voxels in the $\mathrm{x}, \mathrm{y}$, and $\mathrm{z}$ direction was calculated for each region in each hemisphere (see Table1). Our registration method provides significantly more focused maps $\left(F(1,5)=21.176, \rho=.006, \eta^{2}=.809\right)$, spanning an average of $8 \mathrm{~mm}$ less in each dimension, with no significant difference among the dimensions.

\subsection{Validation}

Probability maps of Te1.0, Te1.1, and Te1.2 were Table 1. Estimated mean created for each of the fMRI subjects, and shown below is subject one (Fig. 5) . Summary statistics (Eqn. 2) derived from the original [6] and custom probability maps were evaluated using a repeated-measures ANOVA with three factors: probability map type (original or custom), hemisphere (left or right), and subregion of Te1 (Te1.0, Te1.1, or Te1.2). Of the main effects, only the effect of subregion was sigspanning distance $(\mathrm{mm})$ of previous and custom probability maps in each axial direction

\begin{tabular}{|l|c|c|c|}
\hline & $\mathrm{x}$ & $\mathrm{y}$ & $\mathrm{z}$ \\
\hline previous & 28 & 29 & 30 \\
custom & 24 & 20 & 19 \\
\hline
\end{tabular}
nificant $\left(F(2,7)=18.43, \rho=.002, \eta^{2}=.84\right)$ OR $\left(F(2)=28.016, \rho=.000, \eta^{2}=.778\right)$, although the effect of hemisphere was marginally significant $(\rho=.07)$. A significant interaction between probability map type and subregion (see Fig. 6) was observed; no other interactions reached statistical significance.

Sidak-corrected pairwise comparisons were used to examine the main effect of subarea. This revealed that lateral area Te1.2 and middle area Te1.0 did not differ in activity levels, but that both were significantly more active than medial area Te1.1 $(\rho<.005)$. The interaction between subregions of Te1 and probability

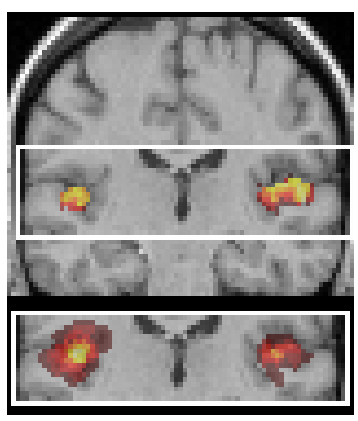

(a) Te 1.0

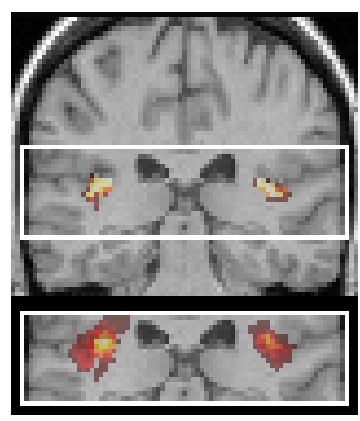

(b) Te 1.1

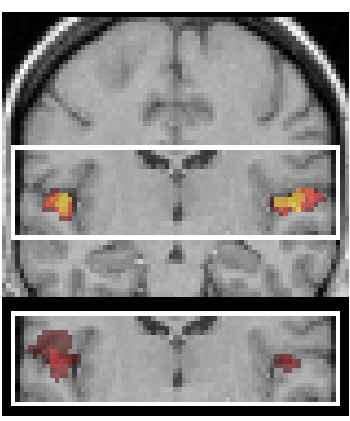

(c) Te 1.2

Fig. 5. Probability maps generated (top) and corresponding section of previous 6 probability maps (bottom), superimposed on subject one's structural 


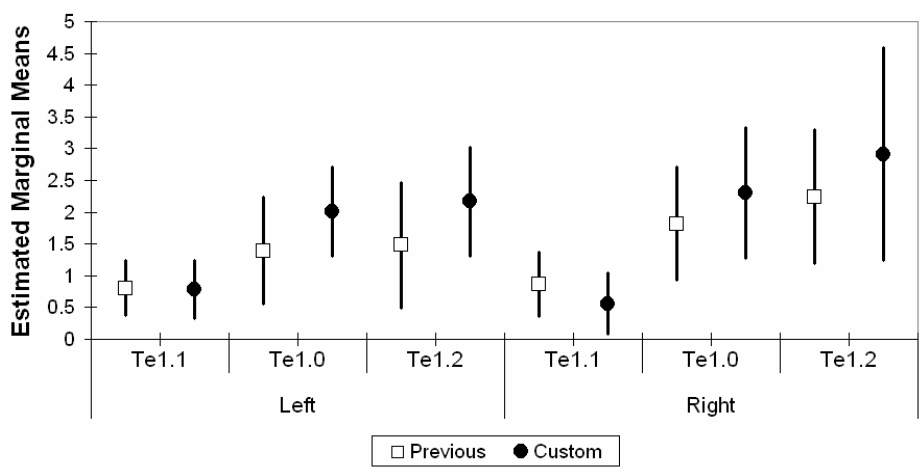

Fig. 6. Estimated marginal means of summary statistics for previous and custom probability maps

map type was also investigated using pairwise comparisons. The custom maps yielded significantly greater activity in area Te1.0 than did the published maps $(\rho=.035)$; the difference between map types was not significant in the other two areas.

Thus, the custom maps do appear to give increased sensitivity to signal change. Furthermore, this analysis demonstrates that Te1.2 and Te1.0 are both sensitive to the presence of temporal structure and pitch in sound, while Te1.1 is not sensitive to this feature. Finally, there is no evidence for hemispheric asymmetry in sensitivity to pitch in these data.

\section{Discussion and Conclusions}

The probability maps we generated for the standard Colin27 brain were significantly more focused than the published ones, indicating that our registration method is superior when precise hypothesis concerning structural-functional relationships in a well defined brain region should be tested. Furthermore, this registration method can be applied to any high-resolution structural image, allowing customised registration of any cytoarchitectonic data with the anatomy of individual fMRI volunteers.

As evidenced by Fig. 4 and [12, deformable registration is superior to affine registration techniques used by Morosan et al. 6], yet the limitation of the technique is speed. The increased number of parameters results in significantly increased registration time: it requires 2.5 days to register both hemispheres of each postmortem to the target fMRI subject, using a $2.6 \mathrm{GHz} \mathrm{P} 4$ with $1 \mathrm{~GB}$ RAM. Future work will involve parallelization and optimization of the registration parameters.

The success of the registration does not depend solely on the method and dimension of parameters used, but also on the inherent variability in structural morphology [12. The use of probability maps registered to a standard brain 
increases the effect of inter-subject variability compared to the use of maps customised for a given subject's anatomy.

We show that such custom probability maps can be used for the analysis of fMRI data. This has several advantages. First, the fMRI data need not be spatially smoothed; essentially, the probability maps themselves acted as filters, yielding a probability-weighted estimate of average activity over a given cytoarchitectonic region. This permits analysis of functional specialization within small, physically adjacent, cortical fields. Our method also permits quantitative comparison of activity in homologous regions in the two hemispheres. Given hemispheric morphological asymmetries, it is not enough to assess signal in homologous voxels, which is how such comparisons are usually accomplished. Our method accounts for morphological asymmetries automatically, and can therefore be used to measure functional laterality accurately and objectively.

The results of the fMRI analysis indicate that lateral region Te1.2 and medial region Te1.0 are sensitive to the presence of pitch in a noisy stimulus. This is highly consistent with neurophysiological investigations in marmosets, which reveal that neurons in a region on the border between A1 and RT (the cytoarchitectonic homologues of human Te1.0 and Te1.2, respectively) are sensitive to pitch [13. However, contrary to previous authors who have suggested a righthemisphere dominance for pitch sensitivity [14], we find no differences between hemispheres in activation levels in homologous regions.

One shortcoming of the fMRI dataset analysed here is that the structural resolution is $2 \times 2 \times 2 \mathrm{~mm}$, which is relatively low. This may have had a negative impact on the resulting probability maps (Fig. 5) when compared to the probability maps for Colin27 (Fig. 4) which has a higher spatial resolution (1x1x1mm). Future work will include probability-map analysis of auditory fMRI datasets with structural acquisitions of $1 \times 1 \times 1 \mathrm{~mm}$.

\section{References}

1. Patterson, R., Uppenkamp, S., Johnsrude, I., Griffiths, T.: The processing of temporal pitch and melody information in auditory cortex. Neuron 36, 767-776 (2002)

2. Amunts, K., Zilles, K.: Advances in cytoarchitectonic mapping of the human cerebral cortex. Neuroimaging Clin N Am 11, 151-169 (2001)

3. Zilles, K., Palomero-Gallagher, N., Grefkes, C., Scheperjans, F., Boy, C., Amunts, K., Schleicher, A.: Architectonics of the human cerebral cortex and transmitter receptor fingerprints: reconciling functional neuroanatomy and neurochemistry. Euro. Neuropsychopharmacology 12, 587-599 (2002)

4. Felleman, D., Van Essen, D.: Distributed hierarchical processing in the primate cerebral cortex. Cereb. Cortex 1, 1-47 (1991)

5. Passingham, R., Stephan, K., Kotter, R.: The anatomical basis of functional localization in the cortex. Nat. Rev. Neurosci. 3, 606-616 (2002)

6. Morosan, P., Rademacher, J., Schleicher, A., Amunts, K., Schormann, T., Zilles, K.: Human primary auditory cortex: Cytoarchitectonic subdivisions and mapping into a spatial reference system. NeuroImage 13, 684-701 (2001)

7. Schleicher, A., Amunts, K., Geyer, S., Morosan, P., Zilles, K.: Observerindependent method for microstructural parcellation of cerebral cortex: A quantitative approach to cytoarchitectonics. Neuroimage 9, 165-177 (1999) 
8. Zilles, K., Schleicher, A., Palomero-Gallagher, N., Amunts, K.: Quantitative analysis of cyto- and receptorarchitecture of the human brain. In: Brain Mapping: The Methods 2nd edn., pp. 573-602 (2002)

9. Rademacher, J., Morosan, P., Schormann, T., Schleicher, A., Werner, C., Freund, H., Zilles, K.: Probabilistic mapping and volume measurement of human primary auditory cortex. Neuroimage 13, 669-683 (2001)

10. Penhune, V.B., Zatorre, R.J., MacDonald, J.D., Evans, A.C.: Interhemispheric anatomical differences in human primary auditory cortex: Probabilistic mapping and volume measurement from magnetic resonance scans. Cereb. Cortex 6, 661-672 (1996)

11. http://www.fil.ion.ucl.ac.uk/spm/software/spm2/

12. Crivello, F., Schormann, T., Tzourio-Mazoyer, N., Roland, P.E., Zilles, K., Mazoyer, B.M.: Comparison of spatial normalization procedures and their impact on functional maps. Human Brain Mapp. 16, 228-250 (2002)

13. Bendor, D., Wang, Q.: The neuronal representation of pitch in primate auditory cortex. Nature 436, 1161-1165 (2005)

14. Zatorre, R.J.: Finding the missing fundamental. Nature 436, 1093-1094 (2005) 